\title{
EFEITO DA QUALIDADE DO VAPOR NA PRODUTIVIDADE DE RESERVATÓRIOS DE PETRÓLEOS ULTRAVISCOSOS
}

\author{
R. G. TEIXEIRA ${ }^{1}$, C. E. de M. JERONIMO², P. H. E. EVARISTO ${ }^{1}$ \\ ${ }^{1}$ Universidade Potiguar. Graduandos em Eng. Petróleo e Gás. \\ ${ }^{2}$ Universidade Potiguar. Professor Doutor do Mestrado em Eng. Petróleo e Gás. \\ E-mail para contato: ponto_rodrigo@hotmail.com
}

\begin{abstract}
RESUMO - A influência da qualidade do vapor (título) utilizado na recuperação avançada de reservatórios de petróleo com elevados níveis de viscosidade é uma das principais variáveis de controle na qualidade do processo de injeção e elemento primordial na apropriação dos custos inerentes a esse processo. Neste trabalho foi desenvolvido um modelo para predição dos efeitos da qualidade da água utilizada na produção do vapor mediante a correlação com dados de monitoração de parâmetros rotineiros (sílica, carbonatos e condutividade). Para estimativa dos ganhos associados a produtividade do reservatório utilizou-se de simulações realizadas no software STARS ${ }^{\circledR}$. Os resultados obtidos apontam para uma boa correlação entre o parâmetro da concentração de sílica na previsão dos valores da qualidade do vapor, porém, sem representar os fenômenos conjugados de deposição, incrustação e corrosão. Sendo assim, o modelo complexo utilizando as três fases, e combinados a concentração especifica dos três principais parâmetros foi desenvolvido, obtendo-se um coeficiente de correlação da ordem de $95 \%$ e um teste positivo para predição, por meio da análise de variância (ANOVA). Diante desses resultados, pode-se utilizar esses modelos como ferramentas para prever os tempos de parada e perdas na produção, conforme demonstrado no desenvolvimento do trabalho.
\end{abstract}

\section{INTRODUÇÃO}

O petróleo é uma substância oleosa, inflamável, menos densa do que a água, com cheiro característico e cor variando entre o preto e o castanho escuro. Acumulam-se em terrenos chamados bacias sedimentares, formadas por camadas ou lençóis porosos de areia, arenitos ou calcários, formando jazidas. Ali são encontrados o gás natural, na parte mais alta, e petróleo e água nas mais baixas. Sabe-se, também, que para cada jazida de petróleo existem determinadas características que classificam a melhor maneira de como esse óleo deve ser tratado e explorado (Thomas, 2001).

A elevada viscosidade de alguns dos petróleos pesados faz com que a produção primária seja muito pequena, e com o declínio das reservas de "óleos convencionais", as atenções da indústria petrolífera se voltam para a recuperação avançada de óleos pesados (API entre 10 e 20), sendo a injeção de vapor um dos mais usados (Viana, 2008). Essa técnica para óleos pesados e viscosos 
facilita a exploração do mesmo, sabendo que para sua extração é necessário aumentar a temperatura para que a viscosidade diminua e a vazão aumente.

O vapor usado nas técnicas de injeção é produzido por um equipamento chamado Gerador de Vapor $(\mathrm{GV})$ que funciona com uma pressão superior à atmosférica, produzindo vapor a partir da energia térmica de uma fonte qualquer. Nela existem vários equipamentos que são usados para obter um maior rendimento térmico e com maior segurança. Para produção desse vapor, faz-se necessário o consumo significativo de água tratada e praticamente isenta da concentração de sais e outros agentes. Segundo (Gabardo e Naveira, 2007) o uso de vapor em campos no Ceará (Fazenda Belém) e no Rio Grande do Norte (Alto do Rodrigues), é realizado com o emprego da água produzida do próprio processo como alternativa como fonte de fornecimento da água, o que torna o processo mais vulnerável a presença de sais na água.

A água fornecida para esses GVs deve passar por diversos tratamentos antes de virar vapor, principalmente considerando o risco de salinidade associado. Esse processo de tratar a água é de extrema importância para a qualidade do vapor (Titulo), que por sua vez ira influenciar na recuperação do óleo ultraviscoso. De nada adianta a instalação de um equipamento ultramoderno, com todos os acessórios disponíveis e automatizados se não é levada em consideração a qualidade da água e o tratamento químico aplicado (Trovati, 2012).

Uma vez não bem tratada, essa água poderá gerar diversos problemas na tubulação e no reservatório de óleo, afetando assim a produtividade do campo a ser explotado. A alimentação de água com boa qualidade, no entanto, elimina, antecipadamente, grande parte dos problemas que normalmente ocorrem em geradores de vapor. Cientes de todos os detalhes mencionados, consideremos o modelo ideal para geração de vapor uma água com as seguintes características: menor quantidade possível de sais e óxidos dissolvidos, ausência de oxigênio e outros gases dissolvidos, isenta de materiais em suspensão, ausência de materiais orgânicos, temperatura elevada e $\mathrm{pH}$ adequado.

Considerando-se o impacto da qualidade da água na geração de vapor, e consequentemente, no campo produtor, esse trabalho consiste no desenvolvimento de estudos para analisar a influência da qualidade da água no título do vapor e nas condições de produção do reservatório, por meio de simulações matemáticas dos níveis de entalpia, fatores de previsibilidade de incrustações e riscos de degradação dos meios porosos. A fim de elaborar um modelo que utilize dos parâmetros do controle da qualidade da água para previsibilidade dos impactos das incrustações no título do vapor obtido e nos níveis de produção dos reservatórios associados.

\section{MATERIAIS E MÉTODOS}

\subsection{Universo/Amostra da Pesquisa}

A área de abrangência do trabalho foram pesquisas bibliográficas referentes a produção de vapor, tratamento da água produzida de campos de petróleo e os modelos de predição de fenômenos de corrosão e incrustação. Bem como, foram obtidos dados do controle da qualidade da água 
produzida para campos de produção de petróleo realizado por empresa especializada, atuando em diferentes estados do Brasil (RN, ES, SE e AL). Foram coletadas informações quanto as concentrações de sílica e carbonatos, e os níveis de condutividade da água utilizada. Além dos resultados do título do vapor produzido.

Os dados utilizados para avaliar características de reservatórios e impactos da qualidade da água serão secundários, oriundos de pesquisas desenvolvidas por bases de pesquisas potiguares, acerca da bacia explorada nos estados do Rio Grande do Norte.

\subsection{Plano de coleta de dados}

As etapas para desenvolvimento da pesquisa foram elencados conforme segue:

1. Levantamento bibliográfico das técnicas de tratamento de água para produção de vapor, entraves operacionais e o controle da qualidade associado ao processo;

2. Obtenção de dados correlatos para a qualidade da água, modelos de predição da incrustação de águas, impactos nas deficiências térmicas na produção do vapor e qualidade do vapor;

3. Caracterização de um reservatório de petróleo e a influência da qualidade do vapor na produtividade dos poços associados a um processo de injeção de vapor;

4. Modelagem e simulação dos fenômenos de produção com os diferentes níveis de incremento do título do vapor no reservatório.

5. Correlação dos dados obtidos entre qualidade do vapor, água produzida tratada e produtividade dos reservatórios de campos maduros para petróleos ultra-viscosos.

O tratamento dos dados coletados foi qualitativo com subsídios do tratamento quantitativo, ou seja, quali-quantitativos. Para analisar os dados coletados através das pesquisas será utilizada a análise de conteúdo. Para tratamento dos modelos desenvolvidos realizou-se análises estatísticas baseadas nos coeficientes de determinação dos ajustes dos modelos e da análise de variância, com uso dos recursos do Microsoft Excel®.

\subsection{Modelagem e Simulação do Reservatório}

O processo foi realizado através de um simulador comercial, STARS (Steam, Thermal, and Advanced Processes Reservoir Simulator) - versão 2007, um simulador numérico trifásico de múltiplos componentes da CMG (Computer Modelling Group) desenvolvido com a finalidade de simular recuperações térmicas de óleo.

O efeito do vapor foi analisado por meio das técnicas de injeção contínua e cíclica, em diversas temperaturas, com intuito de avaliar a capacidade dessa configuração para transferir calor ao reservatório em diferentes situações, em especial para os níveis de título de vapor obtidos nos 
diferentes níveis de qualidade da água. As propriedades do reservatório foram utilizadas conforme sugeridos por Medeiros et al (2011).

\subsection{Modelagem da Qualidade do Vapor}

Para previsão da qualidade do vapor, desenvolveu-se um modelo considerando o efeito das incrustações. Nesse emprega-se resultados de cinética química da deposição de sais, que simultaneamente fornece o ponto em que o vapor gera o menor impacto na transferência para a fase sólida. O modelo foi desenvolvido através da adaptação de Bombardelli et al (2004).

De Acordo com o esquema da Figura 1, tem-se que A, B e C são respectivamente a Deposição física, Incrustação e Corrosão Química, e k1, k2 e k3 representa a velocidade especifica que cada um desses fenômenos ocorre durante o processo de geração do vapor no sistema. Sendo assim, foi feita uma relação na tentativa de correlacionar tais mecanismos a qualidade do vapor obtido, esse esquemático é demonstrado na equação (4).

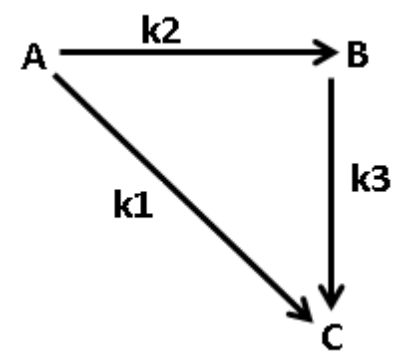

Figura 1 - Esquema de Incrustação e Corrosão

$$
\begin{gathered}
(\boldsymbol{X})=\frac{k_{1}-k_{3}}{k_{1}+k_{2}-k_{3}} e^{-\left(k_{1}+k_{2}\right) C e}+\frac{k_{2}}{k_{1}+k_{2}-k_{3}} e^{-k_{3} C e} \\
t_{1}=\frac{1}{k_{1}+k_{2}} \quad t_{2}=\frac{1}{k_{3}}
\end{gathered}
$$

Onde: $\mathrm{A}_{1}=\frac{\mathrm{k}_{1}-\mathrm{k}_{3}}{\mathrm{k}_{1}+\mathrm{k}_{2}-\mathrm{k}_{3}} \quad, A_{2}=\frac{k_{2}}{k_{1}+k_{2}-k_{3}}$

Os valores relacionados no citado modelo busca relacionar os principais componentes numa única variável, que neste trabalho foi chamado de $\mathrm{Ce}$, que corresponde a relação entre Sílica, Carbonato e Condutividade Térmica com o composto químico do Aço Carbono como mostra a equação: $C e=((50 / 0,3) *$ Sílica $+(50 / 0,5) *$ Carbonato $+(50 / 0,3) *$ Condutividade $) / 305,13$ 
Para se chegar a essa equação foi considerado o peso ponderado da incrustação de cada composto químico e do tubo limpo, juntamente com a média da condutividade térmica de cada composto, de acordo com Kurita (2014). O ajuste dos dados experimentais foi realizado mediante o modelo expdec2, do software Origin 8.1.

\section{RESULTADOS E DISCUSSÕES}

Fazendo-se um estudo da correlação entre esses parâmetros e a sua influência nos níveis da qualidade do Vapor na Figura 2, é apresentado um gráfico de dispersão que relaciona o Título e a concentração de Sílica da água alimentada. Observou-se um nível de confiabilidade de 95\% com uma relação exponencial entre as duas variáveis. Entretanto o uso desse modelo como única forma de predição dos eventos de incrustação torna-se bastante vulnerável e limitada, visto que o mesmo não teve aprovação quando submetido a um teste de análise de variância (ANOVA), sendo assim, faz-se necessário o desenvolvimento de modelos mais complexos e robustos para garantir a previsibilidade desses eventos. O mesmo comportamento não foi observado para o nível de concentração de carbonatos, onde a correlação apresentou um coeficiente de determinação inferior a 80\%.
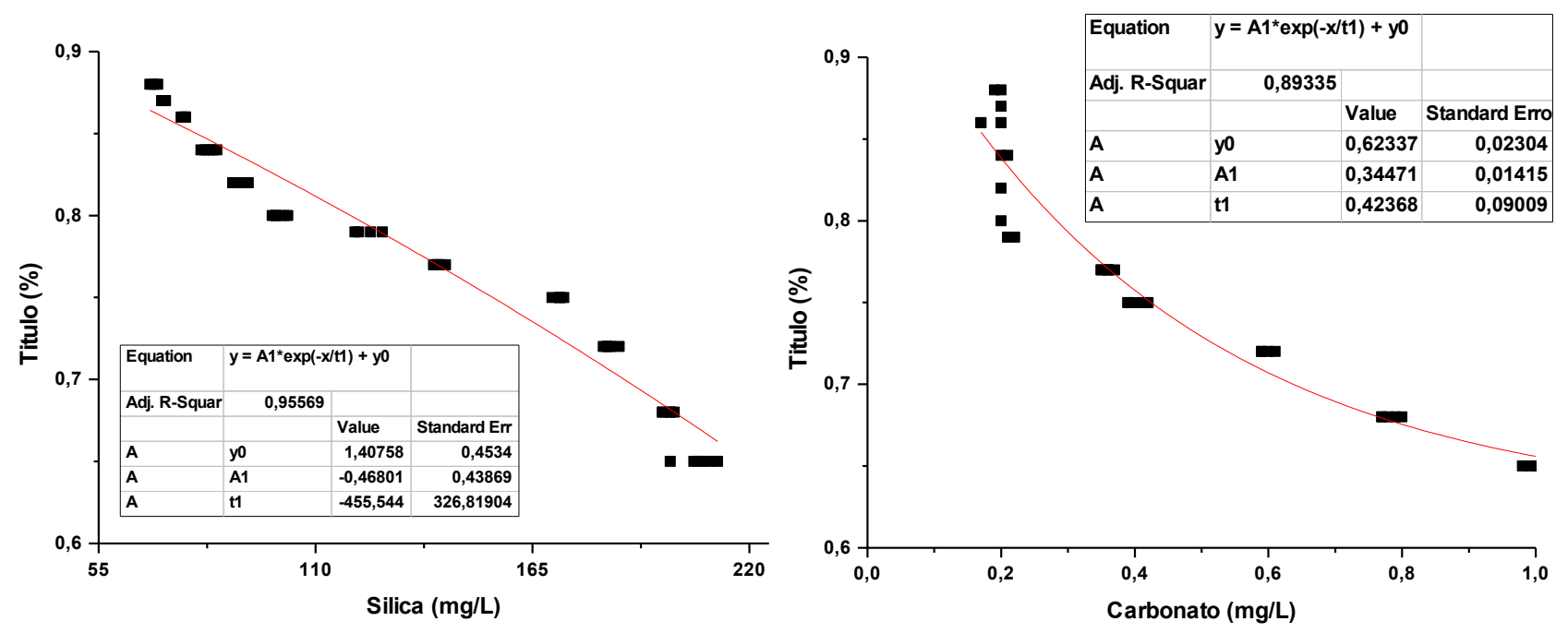

Figura 2 - Modelo de dispersão entre a Concentração de Sílica e Carbonatos na Água e o Título do vapor produzido.

Como uma alternativa de conjugar os parâmetros mais importantes no controle da qualidade do vapor (titulo) foi desenvolvido um modelo para ajustar esses em uma única equação, conforme descrito no tópico de metodologia deste trabalho. O ajuste do modelo proposto aos dados experimentais é apresentado na Figura 3. O referido ajuste apresenta um coeficiente de determinação de $95 \%$ de confiabilidade para os dados experimentais modelados. Para validar o modelo e garantir a predição deste, foi feita uma análise de dados entre os títulos obtidos experimentalmente e os calculados pelo modelo, no qual foi obtido um $\mathrm{F}=0,89366$ que consolida a validade do modelo de 
acordo com a referência. Na Tabela 1 são apresentados os resultados do tratamento estatístico, que valida o caráter preditivo do modelo.

Tabela 1 - Resultados da Análise de Variância (ANOVA)

\begin{tabular}{|l|l|l|l|l|}
\hline Fonte da Variância & SQ & GL & MQ & F \\
\hline Entre Grupos & 0,003955 & 1 & 0,003955 & 0,89366 \\
\cline { 1 - 4 } Dentro dos Grupos & 0,628461 & 142 & 0,004426 & \\
\cline { 1 - 4 } Total & 0,632416 & 142 & & \\
\hline
\end{tabular}

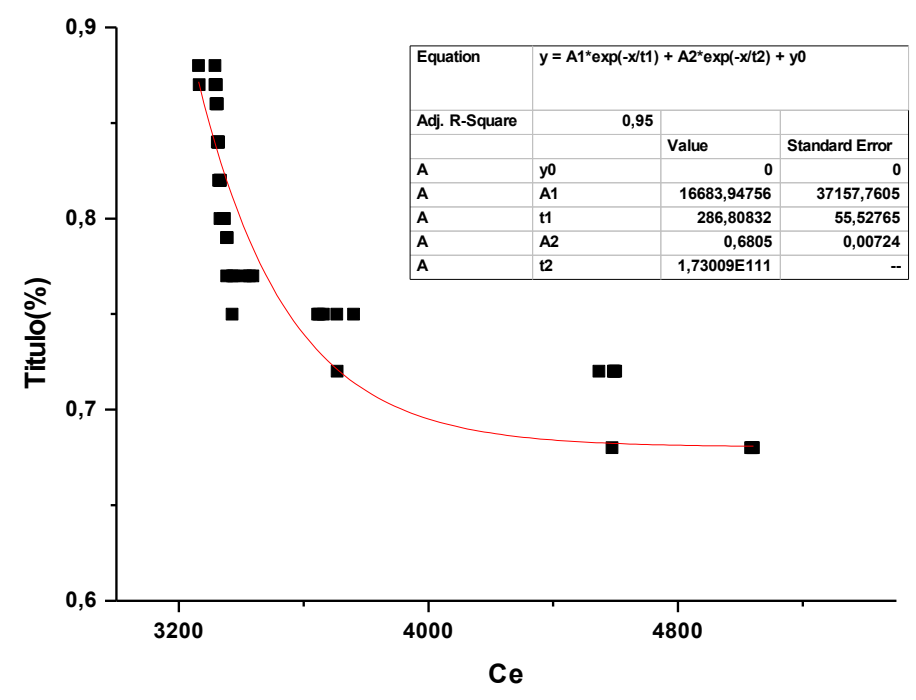

Figura 3. Modelo Combinados dos parâmetros de qualidade da água na predição dos valores do título do vapor produzido.

Esse efeito do título do vapor foi simulado nas características de produtividade do reservatório, e são apresentados na Figura 4. Nessas, observa-se a forte tendência de declínio para introdução do vapor com níveis reduzidos de título, consequentemente de energia. 



Figura 4. Gráfico do volume de Óleo acumulado em relação aos meses, em função do nível da qualidade do vapor injetado.

\section{CONCLUSÕES}

Com base nos resultados obtidos neste trabalho é possível concluir que o modelo do STARS e o formato idealizado para predição dos valores de título são satisfatórios para estimativa de ganhos e perdas do processo, com as oscilações da qualidade da água e consequentemente no impacto provocado no título do vapor produzido. Num nível de 95\% de confiabilidade os modelos apresentaram-se com características preditivas e validados por uma análise de variância. Entretanto, a proposta pode servir para validar um mecanismo genérico de previsibilidade, porém, deve-se respeitar as características especificas dos reservatórios quanto as características do petróleo e da água produzida.

\section{REFERÊNCIAS}

ANDRADE; Maria Margarida de. Introdução à Metodologia do Trabalho Cientifica: elaboração de trabalhos na graduação. 6 ed. São Paulo: Atlas,2003.

BOMBARDELLI, C. et al. Análise paramétrica de um modelo proposto para a incrustação em equipamentos de destilação. Instituto Brasileiro de Petróleo e Gás - IBP. $3^{\circ}$ Congresso Brasileiro de P\&D em Petróleo e Gás. 2004.

CERVO, Amaro L.; BERVIAN, Pedro A. Metodologia científica. 5 ed. São Paulo: Prentice Hall, 2002.

COELHO, L.M.R., "Simulação Numérica de Tecnologias Limpas na Queima de Carvão 
Pulverizado", Documento provisório de Tese de Doutoramento, Universidade técnica de Lisboa, Portugal, 2004.

COELHO, P. J. (a), "Mathematical Modeling of the Convection Chamber of a Utility Boiler - The Theory", Numerical Heat Transfer Part A - Applications, vol. 36, pp.411-428, 1999.

COELHO, P. J.(b), "Mathematical Modeling of the Convection Chamber of a Utility boiler - An Application", Numerical Heat Transfer Part A - Applications, vol. 36, pp.429-447, 1999.

FIGUEIREDO, Antônio Macena de; SOUZA, Soraia Riva Goudinho de. Como elaborar projetos, monografias, dissertações e teses: da redação à apresentação do texto final. 4 ed. Rio de Janeiro: Lumen Juris, 2011.

KEY, J.M., NEDDERMAN, R.M., Fluid mechanics and transfer process, Cambridge University Press, Cambridge, 436 p., 1985.

KURITA. Manual de operação de Caldeiras. Ed. Kurita. São Paulo, SP. 2014.

MATTAR, João. Metodologia cientifica na era da informática. 3 ed. São Paulo: Saraiva, 2008.

MEDEIROS, E., et al.. ANÁLISE DE DIFERENTES VISCOSIDADES A PARTIR DA APLICAÇÃO DO AQUECIMENTO GERAL INDIRETO COMO MÉTODO DE RECUPERAÇÃO EM RESERVATÓRIOS PETROLÍFEROS. HOLOS - ISSN 1807-1600, Natal, 5, dez. 2011. Disponível em: <http://www2.ifrn.edu.br/ojs/index.php/HOLOS/article/view/736>. Acesso em: 05 Mar. 2014.

TROVATI, Joubert. Tratamento de água para geração de vapor: caldeiras. Disponível em: $<$ http//www.snatural. com.br/PDF/Torre-Caldeira-Tratame com.br/PDF/Torre-Caldeira-TratamentoAgua-caldeira.pdf $>$ 